\title{
Notfallteams müssen früher intervenieren
}

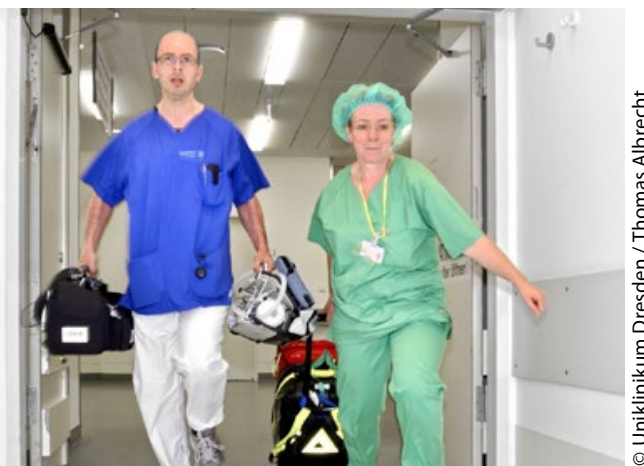

Notfallteam: Ines Reichel und Dr. Andreas Güldner auf dem Weg zu einem Patienten.

— In deutschen Kliniken kommt es jedes Jahr zu rund 93.000 Herz-Kreislaufstillständen. In $80 \%$ der Fälle treten schon Stunden vorher Symptome auf, die auf einen drohenden Herz-Kreislauf-Stillstand hindeuten. Wenn bereits zu diesem Zeitpunkt das Notfallteam gerufen wird, lässt sich das Risiko der lebensbedrohlichen Krisen verrin- gern. Allerdings wird lediglich ein Fünftel aller in deutschen Krankenhäusern tätigen innerklinischen Notfallteams (Medical Emergency-Teams - MET) bereits bei Warnsymptomen aktiv. Das ist das Ergebnis einer von der Klinik für Anästhesiologie und Intensivtherapie des Universitätsklinikums Carl Gustav Carus Dresden vorgenommenen Auswertung von Langzeitdaten.

Als eine der Vorreiterkliniken in Deutschland hat das Dresdner Uniklinikum 2012 die Notfallversorgung insbesondere durch deutliche Senkung der Alarmierungsschwelle seines Notfallteams optimiert. Damit konnte die Überlebensrate auf das Dreifache des deutschen Durchschnittswerts gebracht werden. Dank dieses Engagements gehört das Projektteam am Dresdner Universitätsklinikum zu den diesjährigen Trägern des Deutschen Preises für Patientensicherheit.

Damit Ärzte und Pflegepersonal des Klinikums die Anzeichen einer lebensbedrohli- chen Situation zuverlässig erkennen, haben die Anästhesisten einen Kriterienkatalog erstellt und schulen die Mitarbeiter regelmäßig. Dazu gehört auch ein Reanimationstraining: Für jeden Beschäftigten der Pflege besteht die Pflicht, einmal im Jahr einen solchen Kurs zu besuchen. Diese Maßnahmen sind Teil des Konzepts zum interdisziplinären Notfallmanagement, zu dem Vereinheitlichung des Notfallequipments, die Anschaffung automatisierter Defibrillatoren sowie eine verbesserte Logistik der Alarmierung und des Transports gehören.

Die Wirksamkeit der Maßnahmen zeigt sich darin, dass die Zahl der notwendigen Reanimationen trotz steigendem Schweregrad und steigender Zahl an Patienten stabil bleibt: Auch die Rate der reanimierten Patienten, die 30 Tage nach der Entlassung noch leben, ist am Klinikum dreimal so hoch wie im Bundesdurchschnitt.

www.uniklinikum-dresden.de

\section{Broschüre \\ Leben mit Demenzkranken}

— Die Deutsche Alzheimer Gesellschaft hat die Broschüre "Leben mit Demenzkranken. Hilfen für schwierige Verhaltensweisen und Situationen im Alltag" neu aufgelegt. Die Autoren möchten dazu beitragen, den Alltag mit Demenzerkrankten zu erleichtern. Sie betonen, dass es keine Patentrezepte für den Umgang mit Demenzkranken gibt. Aufgezeigt werden Strategien und Anregungen für den Umgang mit den Verhaltensweisen, die von Angehörigen am häufigsten als belastend beschrieben werden. Für $4 €$ kann sie unter https:// shop.deutsche-alzheimer.de/broschueren/33/leben-mit-demenzkranken bestellt werden.

www.deutsche-alzheimer.de

\section{Zeitreise}

\section{Schwesterntrachten auf dem Laufsteg}

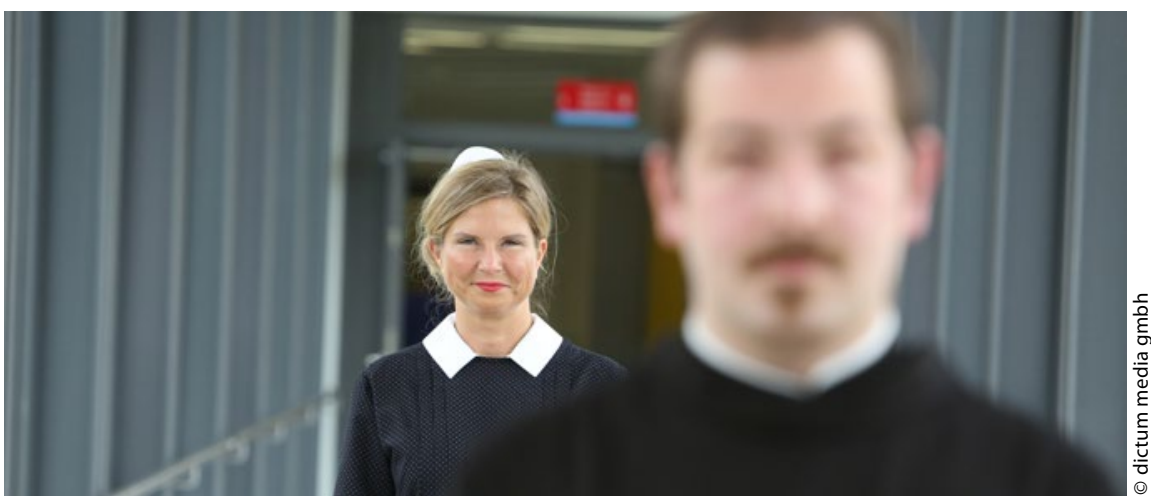

- Das Neurologische Rehabilitationszentrum Godeshöhe e.V. (NRZ) veranstaltete eine außergewöhnliche Modenschau - eine Zeitreise durch die Trachten verschiedener Ordensgemeinschaften und Schwesternschaften. Die Models, die die Ordens- und Schwesternkleider vorführten, kamen nicht von einer Casting-Agentur, sondern arbeiten sonst selbst in der Krankenpflege des Neurologische Rehabilitationszentrum Godeshöhe. Zusammengetragen hat die
Arbeitskleidung aus den unterschiedlichen Epochen Tomislav Kovacevic, selber Gesundheits- und Krankenpfleger beim NRZ Godeshöhe. Seit vielen Jahren beschäftigt er sich mit der Historie der europäischen Krankenpflege und den verschiedenen Schwesternkostümen. Viele der gezeigten Kleidungsstücke waren Originale.

www.godeshoehe.de 